\title{
Approach for preliminary determination of the type and number of vertical rods in grounding installations
}

\author{
Petar Petrov ${ }^{1}$, Georgi Velev ${ }^{1, *}$, and Krasimir Ivanov $^{1}$ \\ ${ }^{1}$ Technical University of Gabrovo, 5300 Gabrovo, Hadzhi Dimitar Str., 4, Bulgaria
}

\begin{abstract}
The paper inhere describes a new practical approach for fast preliminary onsite estimation of the number and the kind of vertical grounding rods as essential components of all grounding systems in electrical power facilities, networks and installations in industry, communal services, mining enterprises, etc.
\end{abstract}

\section{Introduction}

The protective grounding is a main technical measure for protection against electrical shocks intended for class I of electrical devices and consumers in insulated from earth electrical power networks and installations and an additional safety measure along with zero-bonding in directly earthed electrical power networks and installations. Grounding devices are used also as a component of lightning protection systems of buildings and openair facilities and for electrostatic charges protection in underground mines and explosion and fire hazard productions [1].

The maximum permissible values of the transient grounding electrodes' resistance in electrical installations with rated voltage up to $1000 \mathrm{~V}$ and the impulse grounding resistance in lightning protection systems have norm regulated values as defined in the Bulgarian national standards $[1,2]$, all synchronized with the EU and some international regulations $[3,4]$.

The achieving of the desired grounding resistance values is realized by installing grounding systems, which requires design and project activities following a specific methodology. According to [2], the technical condition of grounding installations is supervised on regular basis. In cases of deviations from the required resistance values it is necessary the grounding system to be repaired and renovated in order to improve the transient grounding resistance. Such reconstructions may include: construction of a new grounding system; installation of additional grounding electrodes in order to lower grounding resistance for meeting the requirements.

All this makes topical the following tasks:

- Preliminary estimation of the type and number of vertical grounding rods, options for their installation and repair costs;

- In cases of deviations from the required resistance values - express evaluation of the

*Corresponding author: g.velev@gmail.com 
required number of vertical grounding rods, needed for improvement of grounding resistance and solving the problem. Conclusions about the technical appropriateness of such a decision;

- An approach that gives a preliminary and fast reasonable technical solution about the future design of the grounding system.

\section{Theoretical model of the proposed approach}

Artificial grounding electrodes, which are used in practice, are of special design (vertical grounding electrodes - steel angular winkles or pipes; horizontal grounding electrodes steel bars). The construction of the grounding system requires a design in which are selected the type of grounding electrodes, their location and distribution in order to optimize the system.

In some sources such calculations are made by complex numerical methods, engaging electrical field studies in conducting medium such as earth $[3,5,8]$.

The paper inhere describes a preliminary express approach for assessment of grounding systems being dimensioned at the moment. This approach allows for fast determination of the required number of vertical grounding rods, their location, taking into account their geometrical dimensions and the method for their installation.

In practice, for homogeneous in depth soils it is preferable to use tubular grounding rods because of the following reasons: a high mechanical strength; technical option for mechanized installation on larger depths. Practice has shown that the most suitable for use are tubular grounding rods with diameter $\Phi(20-50 \mathrm{~mm})$ which are mounted at depths $l=2.5-6 \mathrm{~m}$. " $\Gamma$ " shaped steel winkles can also be used as vertical grounding rods but in this case their cross-section dimensions must be converted to tubular vertical rod with equivalent diameter $d$, where $b$ is the winkle's width $[6,7]$ :

$$
d=0.95 \cdot b, \mathrm{~m} \text {. }
$$

The resistance of a single grounding rod is strongly dependent on soil resistivity $\rho$, where the electrode has been installed. For soils with low resistivity grounding rods are with small geometrical dimensions (diameter $d$ and length $l$ ), they are installed in smaller depths and their number is also reduced. Measurements of soil resistivity are essential prior a successful grounding system design in regard with the site where the grounding system will be installed. Also the measured soil resistivity value must be corrected by the season's factor $\varphi$, which takes into account the in-depth influence of soil moisture and temperature on resistivity and respectively on grounding resistance:

$$
\rho_{S}=\varphi \cdot \rho, \Omega \cdot m \text {. }
$$

To determine the resistance of a single vertical rod with a tubular form is used the following analytical expression:

$$
R_{G}=\frac{0.366 \cdot \rho_{S}}{l} \cdot \lg \frac{4 l}{d}, \Omega .
$$

where: $\rho_{S}, \Omega . \mathrm{m}$ - soil resistivity; $d, \mathrm{~m}$ - diameter of the electrode; $l, \mathrm{~m}$ - depth of electrode's installation in soil (electrode length).

Analytical expression (2) can be written in the following form: 


$$
R_{G}=K \cdot \rho_{S}, \Omega
$$

where $K=\frac{0.366}{l} \cdot \lg \frac{4 l}{d}$.

It is obvious that the coefficient $K$ is a function of the installation depth $l$ and the diameter of the electrode $d$.

Table 1 includes pre-calculated values for the coefficient $K$ for the most often used values of electrode's diameter $d$ and depth of installation $l$.

Table 1. Pre-calculated values for the coefficient K.

\begin{tabular}{|c|c|c|c|c|c|c|c|c|c|}
\hline$l, \mathrm{~m}$ & 20 & 25 & 30 & 35 & 40 & 45 & 50 & 55 & 60 \\
\hline 2 & 0.48 & 0.40 & 0.34 & 0.30 & 0.27 & 0.24 & 0.22 & 0.20 & 0.19 \\
\hline 3 & 0.44 & 0.37 & 0.32 & 0.28 & 0.25 & 0.23 & 0.21 & 0.19 & 0.18 \\
\hline 4 & 0.42 & 0.35 & 0.30 & 0.27 & 0.24 & 0.22 & 0.20 & 0.18 & 0.17 \\
\hline 5 & 0.40 & 0.34 & 0.29 & 0.26 & 0.23 & 0.21 & 0.19 & 0.18 & 0.16 \\
\hline
\end{tabular}

In Figure 1 the graphical relationships $R_{G}=f(l)$ for different values of the electrode's diameter and a fixed soil resistivity of $\rho_{S}=100 \Omega . \mathrm{m}$ are built.

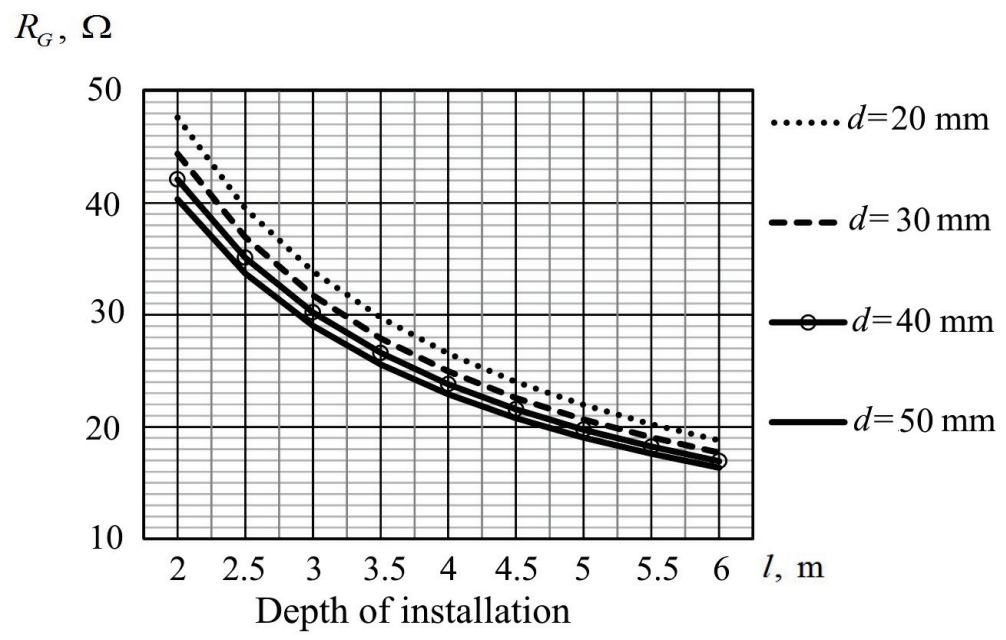

Fig. 1. Graphical dependencies $R_{G}=f(l)$, for different values of the electrode's diameter and a fixed soil resistivity of $\rho_{S}=100 \Omega . \mathrm{m}$.

Soil resistivity values $\rho_{S}$ for different kinds of soils at a frequency $f=50 \mathrm{~Hz}$ have been measured and published in $[3,4,6,7]$. Data is provided for the most often met types of soils with their resistivity given in certain ranges starting from 100 to $1000 \Omega$.m. In this paper it is assumed that intervals of soil resistivity's variation for particular kinds of soils have been preserved, but they are divided into 10 groups, evenly distributed through $100 \Omega$.m. In such case the data for the different kinds of soils $(i=1 . .10)$ is provided and summarized in Table 2.

Analytical expressions (2) and (3) show that the resistance of a single grounding rod is proportional to soil resistivity. The graphical dependencies for grounding resistance of a single rod $R_{G}=f(l)$ in Figure 1 are built for fixed soil resistivity of $\rho_{S}=100 \Omega$.m. 
Table 2. Soil resistivity values of different kinds of soils, divided in 10 groups.

\begin{tabular}{|l|c|c|}
\hline \multicolumn{1}{|c|}{ Type of soil } & number & $\rho_{S}$, \\
& $N_{i}$ & $\Omega . m$ \\
\hline Loam, clay, clay and sand saturated with moisture & 1 & 100 \\
\hline \multirow{2}{*}{ Clay with sand, clay with moisture up to 40\% } & 2 & 200 \\
\cline { 2 - 3 } & 3 & 300 \\
\hline \multirow{2}{*}{ Sand, sand with gravel (wet) } & 4 & 400 \\
\hline \multirow{3}{*}{ Sand, sand with gravel, gravel (dry) } & 5 & 500 \\
\cline { 2 - 3 } & 6 & 600 \\
\cline { 2 - 3 } & 7 & 700 \\
\hline Soils and thin layers of soil less than 1.5 m on a bedrock & 8 & 800 \\
\cline { 2 - 3 } & 9 & 900 \\
\hline
\end{tabular}

For different values of soil resistivity grounding resistance will be proportional to the number of the soil model $N_{i}$, according to Table 2 . Based on these reasons, the graphical relationships of Figure 1 can be presented in the following universal way (Fig. 2) allowing the fast determination of the resistance of a vertical rod with predefined diameter $d$ and depth of installation $l$ for arbitrary value of soil's resistivity $\rho_{S}$ and the corresponding to it number of soil model $N_{i}$ according to Table 2 .

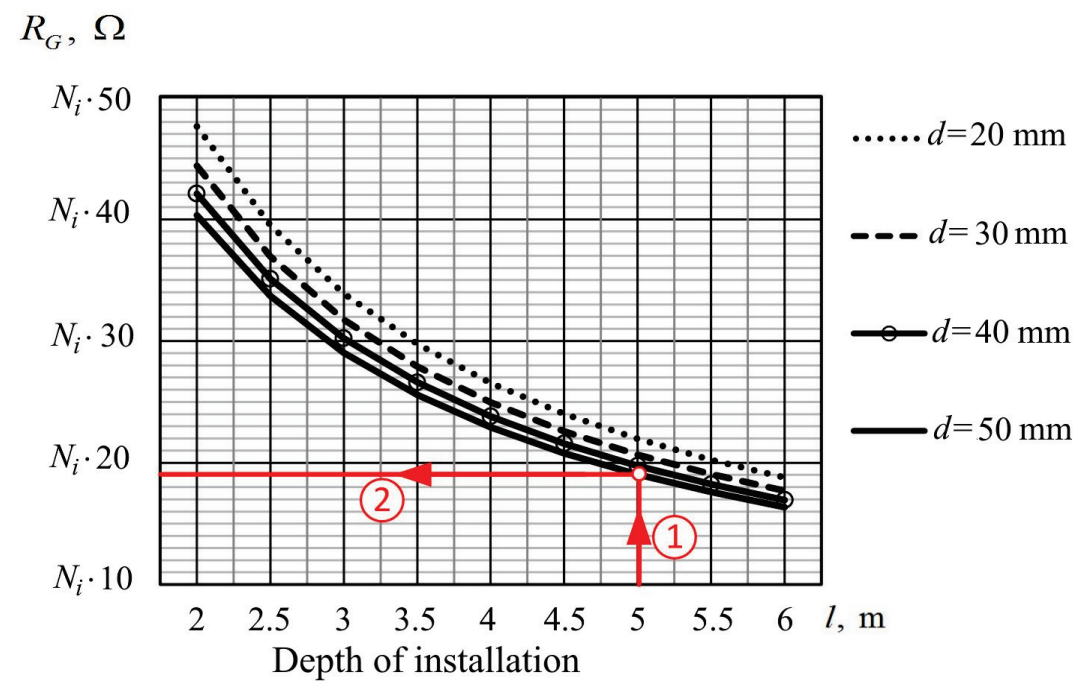

Fig. 2. Universal graphical dependencies $R_{G}=f(l)$, for different values of the electrode's diameter and arbitrary value of soil's resistivity and its corresponding number $N_{i}$.

If the measured soil resistivity is in the intermediate range, the number of the soil model is taken as a decimal fraction e.g. (for $\rho_{S}=140 \Omega . \mathrm{m}, N_{i}=1.4$ ). The presence of the plotted dependencies of Figure 2 makes it possible to select the optimal variant of grounding rods with respect to their geometrical dimensions and the type of soil. This allows direct determination of the resistance of a single grounding rod for a particular soil at the site where the grounding system will be installed.

In the construction of grounding system with identical grounding rods the equivalent grounding resistance $R_{G E}$ is calculated by the analytical expression: 


$$
R_{G E}=\frac{1}{\eta_{V} \cdot \sum_{i=1}^{n} \frac{1}{R_{G}}}, \Omega .
$$

where: $n$ - number of identical vertical grounding rods; $\eta_{V}$ - utilization factor for vertical rods $[6,7]$.

In the design of grounding installations it is relied on reaching an equivalent grounding resistance of the system below the maximum allowable rated value $R_{\text {Grated }}$ as stated into the national, EU and international regulations [1-4].

As a result, it has been obtained an analytical dependence for express calculation of the needed number of identical vertical rods in order to fulfill the requirements:

- The number of vertical electrodes $n_{1}$ is pre-calculated neglecting the mutual influence between them :

$$
-R_{\text {Grated }}=\frac{1}{\sum_{i=1}^{n_{1}} \frac{1}{R_{G}}}=\frac{1}{\frac{n_{1}}{R_{G}}}=\frac{R_{G}}{n_{1}}, \Omega \Rightarrow n_{1}=\frac{R_{G}}{R_{\text {Grated }}} .
$$

- The actual number of vertical electrodes $n$ s obtained by correcting $n_{1}$ with the utilization factor $\eta_{V}$ in dependence on the pre-calculated number of electrodes $n_{1}$, their distribution, length $l$ and the distance between them $a$.

$$
n=\frac{n_{1}}{\eta_{V}}
$$

\section{Numerical example for application of the method}

Calculate the number of vertical tubular grounding rods of a grounding system if the rated grounding resistance of the system is $R_{\text {Grated }}=4 \Omega$. The designed grounding system will be installed with standard tubular rods with length $l=5 \mathrm{~m}$, diameter $d=50 \mathrm{~mm}$, placed in a line at a distance between each other $a=10 \mathrm{~m}$. The measured soil resistivity is $\rho_{S}=100 \Omega . \mathrm{m}$.

1) Measured soil resistivity is corrected with the season coefficient according to (1).

For simplicity we shall assume that the given value $\rho_{S}=200 \Omega . \mathrm{m}$ is already corrected with the season coefficient $\varphi$.

2) The soil model number $N_{i}$ is obtained from Table 2.

For a soil resistivity of $\rho_{S}=200 \Omega . \mathrm{m}$ corresponds a soil model with number $N_{i}=2$;

3) Using Figure 2 for a single vertical electrode with length $l=5 \mathrm{~m}$ and diameter $d=50 \mathrm{~mm}$ is calculated the resistance $R_{G}$ :

$$
R_{G}=N_{i} \cdot 19=2 \cdot 19=38 \Omega .
$$

4) The pre-calculated number of vertical electrodes will be:

$$
n_{1}=\frac{R_{G}}{R_{\text {Grated }}}=\frac{38}{4}=9.5 .
$$


5) The actual number of vertical electrodes $n$ is:

$$
n=\frac{n_{1}}{\eta_{V}}=\frac{9.5}{0.7}=13.5 \approx 14 .
$$

where $\eta_{V}=0.7$ for up to 10 electrodes with length $l=5 \mathrm{~m}$ arranged in a line at a distance between each other $a=10 \mathrm{~m}$.

\section{Conclusions}

The proposed in-here methodology for express determination of the type and number of vertical rods significantly simplifies and relives the work of the designer.

Grounding resistance of a single vertical rod is determined immediately by using Figure 2 depending on soil resistivity and the predefined geometrical dimensions of the electrode - shape, length and diameter.

The calculated resistance of a single vertical grounding rod is used as a base for fast calculation of the necessary number of electrodes needed for reaching an equivalent grounding resistance of the system below the maximum allowable rated value $R_{\text {Grated }}$.

\section{References}

1. Ordinance No. 4 from 22 December 2010 for the Lightning Protection of Buildings, External Facilities and Open Spaces, SG, Issue 6 (Bulgarian standard) (2010)

2. Ordinance No 3 from 9 June 2004 for the Design of Electrical Systems and Power Lines (Bulgarian standard) (2004)

3. IEEE Std 80-2000, IEEE Guide for Safety in AC Substation Grounding, IEEE-SA Standards Board (2000)

4. IEEE Std 81-1983, IEEE Guide for Measuring Earth Resistivity, Ground Impedance, and Earth Surface Potentials of a ground system, American National Standards Institute (1984)

5. S. Stefanov, T. Hristova, L. Atanasov, Potential of an electric field at an arbitrary point in earth, created by different polarity linear and linear and point current sources, Annual of the University of Mining and Geology St. Ivan Rilski, 52, Part III, Sofia, Bulgaria, 29-132, ISSN 1312-1820 (2009)

6. G. Anev, I. Danev, Electrcial Safety in the Open Mines and Industry, PH "Nakra", Radnevo (1996)

7. G. Venkov. P. Petrov, Technical Safety, PH "Gabrovo Print" (2002)

8. Y. L. Chow, M. M. A. Salama, A Simplified Method for Calculating the Substation Grounding Resistance, IEEE Transactions in Power Delivery, 9, No. 2, 736-742 (1994) 